\title{
Innervation of flexor hallucis longus muscle: an anatomical study for selective neurotomy
}

\author{
G. Koch ${ }^{1,2}$, L.R. Cazzato², P. Auloge², B.J. Chiang ${ }^{3}$, J. Garnon², P. Clavert'1,4 \\ ${ }^{1}$ Department of Anatomy, University of Strasbourg, Strasbourg, France \\ 2Department of Interventional Radiology, Strasbourg University Hospital, Strasbourg, France \\ ${ }^{3}$ Department of Radiology and Imaging, Queen Elizabeth Hospital, Kowloon, Hong Kong \\ ${ }^{4}$ Department of Orthopaedic Surgery - CCOM, Strasbourg University Hospital, IIlkirch, France
}

[Received: 18 December 2018; Accepted: 12 January 2019]

\begin{abstract}
Background: The aim of the study was to describe the innervation of flexor hallucis longus (FHL) and obtain its surgical coordinates to facilitate selective neurotomy. Materials and methods: Fifteen embalmed lower limbs of adults were studied. Anatomical dissections to isolate the innervating branches of FHL were performed. Distance between the supplying nerve of FHL, including both its origin and termination, and the medial malleolus were obtained, providing anatomical coordinates beneficial for surgery.

Results: In all cases, FHL was innervated by only one branch, which originated from the tibial nerve. Mean distance between the medial malleolus and the nervous branch origin was $21.39 \pm 3.05 \mathrm{~cm}$. Mean distance between the medial malleolus and the nervous branch termination was $12.7 \pm 1.59 \mathrm{~cm}$. Length of the nervous branch innervating FHL was proportional to the length of the leg, measuring $8.69 \pm 2.45 \mathrm{~cm}$. All nerves were located $15-17.4 \mathrm{~cm}$ above the medial malleolus. Conclusions: This anatomical study traced valuable surgical coordinates useful for performing selective peripheral neurotomy on the nerve branch innervating the FHL. (Folia Morphol 2019; 78, 3: 617-620)
\end{abstract}

Key words: flexor hallucis longus, neurotomy, nerve, hallux claw toe

\section{INTRODUCTION}

Hallux claw toe is a spastic deformity of the great toe characterised by an extension of the first metatarsal joint combined with flexion of the first interphalangeal joint. This deformity is mainly caused by contraction of the flexor hallucis longus (FHL) [4]. This condition occurs in hemiplegic patients after stroke. Chief complaints include pain at the tip of the hallux and under the first metatarsal head that often leads to gait disturbance and painful toe/shoe conflict. The deformity may also lead to devastating skin lesions that are difficult to manage.

Several treatments may be used to manage hallux claw toe. They include medication, physical therapy, and motor point block using botulinum toxin. Medication and physical therapy may not provide sufficient benefit, and may have undesirable systemic effects [3]. Botulinum toxin injection is efficient for management of spasticity, but only for 5-6 months, unable to offer a persistent solution [6]. Almost half of the patients require additional injections to achieve the desired clinical benefit; however, additional injections are not normally recommended because of antibody formation [4]. Historically hallux claw toe has been treated with the Jones procedure or its modification, but complications due to secondary biomechanical alteration across the metatarsophalangeal joint have been reported [5].

Address for correspondence: Dr. G. Koch, Department of Anatomy, University of Strasbourg, 4 rue Kirschleger 67000 Strasbourg, France, tel: +33 3695516 76, fax: +33 3695503 03, e-mail: guillaume.koch@unistra.fr 
A minimally invasive approach for long lasting relief of spasticity of FHL is selective neurotomy by sectioning the nervous branches innervating FHL. To find these branches during surgery without performing an extensive skin incision, their anatomical location must be identified.

This anatomical study is intended to describe the innervation of FHL and obtain its surgical coordinates to facilitate selective neurotomy.

\section{MATERIALS AND METHODS}

All anatomical specimens used for this study were from human cadaver donations to the anatomy department at the authors' institution. The specimens include 15 unpaired (5 left and 10 right) embalmed cadaveric lower limbs, which have been injected with formalin and alcohol solution via the femoral artery prior to amputation. None of the lower limbs demonstrated any evidence of previous surgical procedures, trauma or deformity to the examined regions.

\section{Anatomical dissection}

Anatomical dissection of lower limbs to isolate the innervating branches of FHL and perform various measurements were made to obtain anatomical coordinates for surgery.

Starting from the medial aspect of the popliteal fossa (localised by the tendon of semitendinosus), skin incision was made to extended to the medial side of the ankle following a postero-medial approach along the sural triceps medial edge.

After incising the deep crural fascia of the leg, the flexor muscles in the superficial posterior compartment (soleus, gastrocnemius and plantaris muscles) were exposed. The gastrocnemius, soleus and plantaris muscles were then retracted laterally and the transverse intermuscular septum incised to expose the muscles of the deep posterior compartment. A careful dissection was performed to identify the course, number, point of origin, and muscular inlet of nervous branches for FHL (Fig. 1).

\section{Data collection}

The length of the legs was measured from the most posterior part of the medial tibial plateau and the medial malleolus extremity. The extremity of the medial malleolus was set as an anatomical landmark. Then, two measurements were obtained: the distance between the medial malleolus extremity and the nerve of the FHL termination (defined as medial malleo-

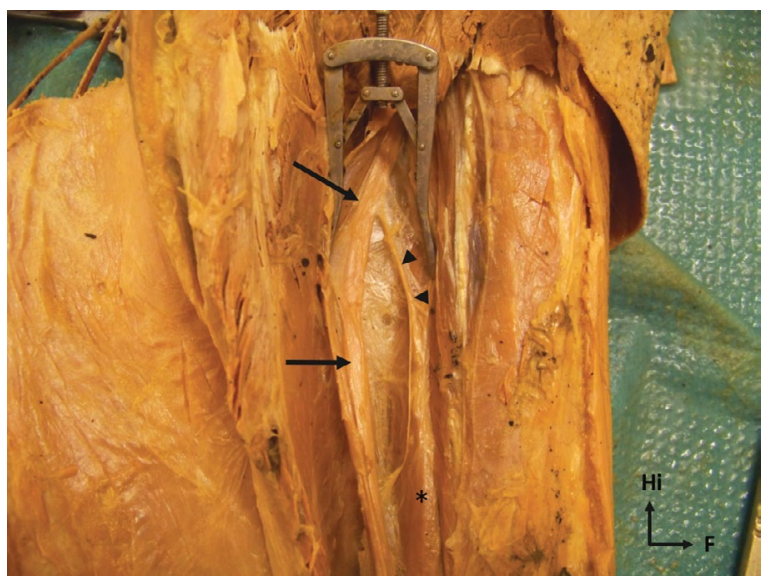

Figure 1. Dissection of a left leg posterior compartment showing nerve branches innervating the flexor hallucis longus (FHL) (arrow heads) arising from the tibial nerve (arrows) and innervating the FHL $(*)$.

lus-nerve termination distance [MTD]); the distance between the medial malleolus extremity and the point of origin of the nerve for the FHL (defined as medial malleolus-nerve origin distance [MOD]). The length of the branch for the FHL was calculated (difference between MOD and MTD).

All measurements were performed by a single operator using callipers (measured in centimetres). Mean, median, standard deviation, minimum and maximum values for each parameter were calculated, collated, and tabulated.

\section{RESULTS}

The mean length of the legs was $35.5 \mathrm{~cm}$ (range $29.5-39 \mathrm{~cm}$ ). In all 15 specimens, the nervous branches for the FHL were found in the deep posterior compartment of the leg. These branches arose from the tibial nerve after it provided branches to the gastrocnemius and soleus muscles and ran along the tibial nerve into the deep posterior compartment between the tibialis posterior and the FHL. The nervous branches for the FHL all entered the muscle at its posterior part. In all the 15 specimens, a unique innervating branch for the FHL was identified: its course and relationships were constant. The mean MTD was $12.7 \mathrm{~cm}$ (range 10-15 cm) and the mean MOD was $21.39 \mathrm{~cm}$ (range 17.4-27 cm). The mean length of the nervous branch for the FHL was $8.69 \mathrm{~cm}$ (median $9 \mathrm{~cm}$, range $4.5-14 \mathrm{~cm}$ ). Length of the nervous branch innervating $\mathrm{FHL}$ was proportional to the length of the leg. Detailed results are summarised in Table 1 and descriptive statistics are detailed in Table 2. 
Table 1. Detailed results in centimetres

\begin{tabular}{lccc}
\hline Specimen & MTD & MOD & Branch for FHL \\
\hline 1 & 15 & 27 & 12 \\
2 & 11.5 & 19 & 7.5 \\
3 & 14.5 & 24 & 9.5 \\
4 & 12 & 26 & 14 \\
5 & 13 & 22 & 9 \\
6 & 12 & 23 & 11 \\
7 & 11 & 18 & 7 \\
8 & 12 & 19 & 7 \\
9 & 10 & 17.4 & 7.4 \\
10 & 14 & 18.5 & 4.5 \\
11 & 15 & 25 & 10 \\
12 & 14.5 & 20 & 5.5 \\
13 & 13 & 22 & 9 \\
14 & 11 & 19 & 8 \\
15 & 12 & 21 & 9 \\
\hline
\end{tabular}

MTD - medial malleolus-nerve termination distance; MOD — medial malleolus-nerve origin distance; $\mathrm{FHL}$ - flexor hallucis longus

Table 2. Descriptive statistics

\begin{tabular}{lccc}
\hline & MTD & MOD & Branch for the FHL \\
\hline Mean [cm] & 12.7 & 21.39 & 8.69 \\
Median [cm] & 12 & 21 & 9 \\
Standard deviation & 1.59 & 3.06 & 2.45 \\
Minimum [cm] & 10 & 17.4 & 4.5 \\
Maximum [cm] & 15 & 27 & 14
\end{tabular}

MTD — medial malleolus-nerve termination distance; MOD — medial malleolus-nerve origin distance; $\mathrm{FHL}$ - flexor hallucis longus

\section{DISCUSSION}

The results of this study show that $\mathrm{FHL}$ is innervated by a single nervous branch which was $8.69 \pm 2.45 \mathrm{~cm}$ long and arises from the tibial nerve after it provided branches to the gastrocnemius and soleus muscles.

Our findings concerning the origin and the trajectory of the FHL nervous branches were consistent with described data in the literature $[1,7,8]$.

For all the studied subjects, we only found a single nervous branch innervating $\mathrm{FHL}$, which is different from previous studies where multiple branches were described. Number of nervous branches range from 1.75 [7] up to 4 branches [7]. This difference might be a result of different definition used for nervous branches. We choose to define the main trunk innervating FHL as the point where the mo- tor nerve first pierced the muscle belly [4], whereas other studies counted all the secondary motor entry points on the same nerve. Apaydin et al. [1] described 2.8 branches for FHL (mean; range 1-6) because they also counted branches arising from the main FHL branch. The differing definition of the studied nerve termination also led to differences between our study and literature findings concerning the position of nervous branch termination (MTD) and FHL nervous branch length. Bodily et al. [2], $139 \pm 30.2 \mathrm{~mm}$ or Apaydin et al. [1] who reported a nerve length of $9.4 \mathrm{~cm}$ but with a very large range of measurements $(1.8-24.2 \mathrm{~cm})$.

Other landmarks have been used to study position of posterior compartment muscles such as the apex of head of fibula [1] or the tibial plateau [2]. However, we considered the medial malleolus as a landmark in determining FHL nervous branch localisation. We consider that this bony landmark can be easily palpable preoperatively to estimate nervous branch position.

The highest nerve termination was located $15 \mathrm{~cm}$ above the medial malleolus. The lowest nerve origin was located $17.4 \mathrm{~cm}$ above the medial malleolus. Hence our study suggests that incision made between 15 and $17.4 \mathrm{~cm}$ above the medial malleolus would have identified all nerves in FHL selective neurotomy.

The small number of dissected legs and their unpaired feature are limitation of this study as it didn't allow us to realize a bilateral and comparative study of the coordinates and the length of these nervous branches. However, this study already suffices to describe a relatively short segment $(2.4 \mathrm{~cm}$ long) where it is possible to find nerve branches for FHL.

\section{CONCLUSIONS}

This anatomical study provided valuable surgical coordinates (15-17.4 $\mathrm{cm}$ above the medial malleolus) useful for performing a selective peripheral neurotomy on the nerve branch innervating FHL.

\section{REFERENCES}

1. Apaydin N, Loukas M, Kendir S, et al. The precise localization of distal motor branches of the tibial nerve in the deep posterior compartment of the leg. Surg Radiol Anat. 2008; 30(4): 291-295, doi: 10.1007/s00276-008-0321-x, indexed in Pubmed: 18283390.

2. Bodily KD, Spinner RJ, Bishop AT. Restoration of motor function of the deep fibular (peroneal) nerve by direct nerve transfer of branches from the tibial nerve: an anatomical study. Clin Anat. 2004; 17(3): 201-205, doi: 10.1002/ca.10189, indexed in Pubmed: 15042567. 
3. Gracies J, Nance P, Elovic E, et al. Traditional pharmacological treatments for spasticity part II: General and regional treatments. Muscle Nerve. 1997; 20(S6): 92-120, doi: 10.1002/ (sici)1097-4598(1997)6+<92::aid-mus7>3.3.co;2-e.

4. Lee JH, Han SH, Ye JF, et al. Effective zone of botulinum toxin a injections in hallux claw toe syndrome: an anatomical study. Muscle Nerve. 2012; 45(2): 217-221, doi: 10.1002/ mus.22263, indexed in Pubmed: 22246877.

5. Lui TH. Flexor hallucis longus tendon to extensor hallucis longus tendon transfer for flexible hallux claw toe deformity: a minimally invasive approach. Foot Ankle Int. 2013; 34(2): 303-306, doi: 10.1177/1071100712467431, indexed in Pubmed: 23413075.
6. Suputtitada A. Local botulinum toxin type A injections in the treatment of spastic toes. Am J Phys Med Rehabil. 2002;81(10): 770-775, doi: 10.1097/01.PHM.0000027043.28847.47, indexed in Pubmed: 12362118.

7. Wongphaet $P$, Chinsethagij K, Suarchawaratana S, et al. Precise localization of motor branching and motor points: a cadeveric study. J Med Assoc Thai. 2005; 88(12): 1884-1891, indexed in Pubmed: 16518990.

8. Yu D, Yin $H$, Han $T$, et al. Intramuscular innervations of lower leg skeletal muscles: applications in their clinical use in functional muscular transfer. Surg Radiol Anat. 2016; 38(6): 675-685, doi: 10.1007/s00276-015-1601-x, indexed in Pubmed: 26707590. 